Pacific Journal of Mathematics

ON THE SEMISIMPLICITY OF GROUP RINGS OF LINEAR 


\title{
ON THE SEMISIMPLICITY OF GROUP RINGS OF LINEAR GROUPS
}

\author{
D. S. PASSMAN
}

In this paper we study the semisimplicity problem for group rings of linear groups. We prove the linear group analog of a result which constitutes part of the solution of the semisimplicity problem for solvable groups. Since all of the necessary group ring lemmas have appeared elsewhere, the work here is strictly group theoretic. We consider the possibility of a linear group being covered by a finite union of root sets of centralizer subgroups.

Let $K[G]$ denote the group ring of $G$ over the field $K$. Probably the most fascinating and difficult question asked about this ring is when is it semisimple, that is when does its Jacobson radical $J K[G]$ vanish. If $K$ has characteristic 0 , then by a result of Amitsur [1] $J K[G]=0$ for all fields $K$ which are not algebraic over the rationals and in all likelihood $K[G]$ is always semisimple. Thus the real interest is now in characteristic $p>0$. At this time there is not even a reasonable conjecture as to the answer here and so it is necessary and important that a large number of special cases be studied. So far the only nontrivial family of groups for which this problem has been solved is in fact the solvable groups, that is the groups of finite derived length. It appears that the next family of interest will be the linear groups since some interesting work in this direction has already appeared in [4]. In this paper we study the semisimplicity problem for linear groups.

Let $G$ be a group and let $H$ be a subgroup. We say that $H$ has locally finite index in $G$ and write $[G: H]=1$.f. if for all finitely generated subgroups $S$ of $G$ we have $[S: S \cap H]<\infty$. We say that $G$ is a $\Delta$-group if $G=\Delta(G)$, that is if all conjugacy classes of $G$ are finite. The result on solvable groups was proved in a series of three papers [3], [2], and [5] and is as follows.

Theorem. (Hampton, Passman, and Zalesskii.) Let $K$ be a field of characteristic $p>0$ and let $G$ be a solvable group. Then $J K[G] \neq 0$ if and only if there exists an element $x \in \ni(G)$ (a certain characteristic subgroup of $G$ ) of order $p$ with $\left[G: C_{G}(x)\right]=$ l.f.

We remark that $\ni(G)$ is a particular normal $\Delta$-subgroup of a solvable group which is defined in [5] and we propose to call it the Zalesskii subgroup of $G$. 
Now let $G$ be a linear group. That is, $G$ is a subgroup of the group of units of $L_{u}$, the ring of $u \times u$ matrices over some field $L$ (which need not be at all related to $K$ ). The main results here are.

Theorem. Suppose $K$ is a field of characteristic $p>0$. Let $G$ be a linear group and let $H$ be a normal solvable subgroup. Then $J K[G] \cap K[H] \neq 0$ if and only if there exists an element $x \in \ni(H)$ of order $p$ with $\left[G: C_{G}(x)\right]=l . f$.

CoRollary. Suppose $K$ is a field of characteristic $p>0$. Let $G$ be a linear group and let $H$ be a normal nilpotent subgroup. Then $J K[G] \cap K[H] \neq 0$ if and only if there exists an element $x \in Z(H)$ of order $p$ with $\left[G: C_{G}(x)\right]=l . f$.

Actually the required group ring lemmas have already been proved in [2] and [5]. The work here is strictly group theoretic.

1. Isolated subsets. Let $G$ be a group and let $H$ be a subgroup. We let

$$
\sqrt{H}=\sqrt[G]{H}=\left\{g \in G \mid g^{m} \in H \text { for some integer } m \geqq 1\right\}
$$

be the root set of $H$. Thus for example if $H \triangleleft G$ then $\sqrt{H}$ corresponds to the set of torsion elements of $G / H$. Certainly $\sqrt{H}$ need not be a subgroup of $G$. We start by listing three trivial observations.

Lemma 1. Let $H, H_{1}, H_{2}, \cdots, H_{k}$ be subgroups of $G$. We have

(i) $\sqrt{H_{1} \cap H_{2} \cap \cdots \cap H_{k}}=\sqrt{H_{1}} \cap \sqrt{H_{2}} \cap \cdots \cap \sqrt{H_{k}}$.

(ii) $\sqrt{H^{g}}=(\sqrt{H})^{g}$ for any $g \in G$.

(iii) if $H \subseteq \sqrt{H_{1}} \cup \sqrt{H_{2}} \cup \cdots \cup \sqrt{H_{k}}$ then $\sqrt{H} \subseteq \sqrt{H_{1}} \cup \sqrt{H_{2}} \cup \cdots \cup \sqrt{H_{k}}$.

Lemma 2. Suppose $G=\bigcup_{1}^{n} \sqrt{\overline{H_{i}}}$ with $H_{i} \triangleleft G$. Then for some $j, G=\sqrt{H_{j}}$ or equivalently $G / H_{j}$ is torsion.

Proof. We proceed by induction on $n$, the case $n=1$ being clear. Suppose the result is true for $n-1$ and we consider $n$.

Given $H_{1}, H_{2}, \cdots, H_{n}$ we define the parameter $r$ of this situation to be the minimum number of $H$ 's whose intersection is equal to $N=H_{1} \cap H_{2} \cap \cdots \cap H_{n}$. Clearly $r$ exists, $r \leqq n$ and we prove the $n$ case by induction on the parameter $r$. Say the numbering is so 
chosen that $H_{1} \cap H_{2} \cap \cdots \cap H_{r}=H_{1} \cap H_{2} \cap \cdots \cap H_{n}$. If $r=1$ then $H_{1} \cong H_{2}$ so $\sqrt{H_{1}} \cong \sqrt{H_{2}}, G=\bigcup_{2}^{n} \sqrt{H_{i}}$ and the $n-1$ case yields the result. We may therefore assume that $r>1$. Set

$$
W=H_{2} \cap H_{3} \cap \cdots \cap H_{r}
$$

so by definition $W>N$ and $H_{1} \cap W=N$. There are two cases to consider according to whether $W / N$ is torsion or not.

Suppose first that $W / N$ is torsion. Let $\bar{G}=G / W$ and let $\bar{H}_{i}=$ $H_{i} W / W \cong \bar{G}$. Clearly $\bar{G}=\bigcup_{1}^{n} \sqrt{\bar{H}_{i}}$. Also for $i=2,3, \cdots, r, \bar{H}_{i}=$ $H_{i} / W$ so $\bar{H}_{2} \cap \bar{H}_{3} \cap \cdots \cap \bar{H}_{r}=\langle 1\rangle$. This clearly implies that

$$
\bigcap_{1}^{n} \bar{H}_{i}=\langle 1\rangle
$$

and that the parameter of this situation is less than $r$. By induction for some $j, \bar{G}=\sqrt{\bar{H}_{j}}$ or equivalently $G / H_{j} W$ is torsion. Now $H_{j} W / H_{j} \cong W /\left(H_{j} \cap W\right)$ and this is a homomorphic image of the torsion group $W / N$. Thus $H_{j} W / H_{j}$ is torsion and hence so is $G / H_{j}$. The result follows in this case.

Finally suppose $W / N$ is not torsion and choose $x \in W$ to correspond to an element of infinite order in $W / N$. Let $h \in H_{1}$ and consider the $n+1$ elements $x, h x, h^{2} x, \cdots, h^{n} x$. Then for each $i$ some power of $h^{i} x$ is contained in some $H_{k}$. Since there are $n+1$ elements and only $n$ subgroups it follows that for two different $i, j$ we have $h^{i} x, h^{j} x \in \sqrt{H_{k}}$ for some $k$. By choosing a sufficiently high power $m$ we can assume that $\left(h^{i} x\right)^{m},\left(h^{j} x\right)^{m} \in H_{k}$. Note that

$$
H_{1} \cap W=N \quad \text { so } \quad\left(H_{1} / N\right)(W / N)=\left(H_{1} / N\right) \times(W / N)
$$

is a direct product. We show first that $k \neq 1$. For suppose $k=1$. Then from $\left(h^{i} x\right)^{m} \in H_{1}$ and $h \in H_{1} \triangleleft G$ we have easily $x^{m} \in H_{1}$. Thus $x^{m} \in W \cap H_{1}=N$ a contradiction since $x$ has infinite order modulo $N$. Thus $k \neq 1$. Now clearly $\left(h^{i} x\right)^{m}=h^{i m} x^{m} g$ for some $g \in N \subseteq H_{b}$ so we have $h^{i m} x^{m} \in H_{k}$ and similarly $h^{j m} x^{m} \in H_{k}$ so if $i>j$ then

$$
h^{(i-j) m}=\left(h^{i m} x^{m}\right)\left(h^{j m} x^{m}\right)^{-1} \in H_{k}
$$

and $h \in \sqrt{H_{k}}$. We have therefore shown that $H_{1} \leqq \bigcup_{2}^{n} \sqrt{H_{i}}$ so $\sqrt{H_{1}} \subseteq \bigcup_{2}^{n} \sqrt{H_{i}}$ and $G=\bigcup_{2}^{n} \sqrt{H_{i}}$. The result follows from the $n-1$ case.

This completes the $r$ induction step and the $n$ case is true for all $r$. Thus the lemma follows by induction on $n$.

Now let $G$ be a linear group so that $G \cong L_{n}$ for some field $L$ and some integer $u$ and we fix this notation throughout the remainder of this section. If $H$ is a subgroup of $G$ we let $\hat{H}$ denote its $L$-linear 
span. Thus clearly $\hat{H}$ is a subalgebra of $L_{u}$. We say that $H$ is a pure subgroup of $G$ if $H=\hat{H} \cap G$ and we let $\mathscr{P}(G)$ denote the set of all such $H$. If $H \in \mathscr{P}(G)$ we use $\operatorname{dim} H$ to denote the $L$-dimension of $\hat{H}$. We list a number of trivial observations.

Lemma 3. Let $G$ be as above.

(i) $H \in \mathscr{P}(G)$ if and only if $H=G \cap R$ for some L-subalgebra $R$ of $L_{u}$.

(ii) $\mathscr{P}(G)$ is closed under conjugation by $G$ and arbitrary intersections.

(iii) If $H_{1}, H_{2} \in \mathscr{P}(G)$ and $H_{1}>H_{2}$ then $\operatorname{dim} H_{1}>\operatorname{dim} H_{2}$.

Let $H$ be a pure subgroup of $G$. We say that $H$ is root reduced if

$$
\sqrt{H} \leqq \sqrt{H_{1}} \cup \sqrt{H_{2}} \cup \cdots \cup \sqrt{H_{k}}
$$

for finitely many pure subgroups $H_{i}$ of $G$ implies that $H_{i} \supseteqq H$ for some $i$.

Lemma 4. Let $H \in \mathscr{P}(G)$. Then

$$
\sqrt{H}=\sqrt{H_{1}} \cup \sqrt{H_{2}} \cup \cdots \cup \sqrt{H_{k}}
$$

for finitely many pure root reduced subgroups $H_{i} \subseteq H$.

Proof. We proceed by induction on $\operatorname{dim} H$. If $\operatorname{dim} H=1$ then clearly $H$ is contained in all pure subgroups of $G$. Thus $H$ is certainly root reduced and the result follows here. Suppose now that $\operatorname{dim} H>1$ and that the result is true for all pure subgroups of smaller dimension. If $H$ is root reduced the result is clear so we may suppose not. Then

$$
\sqrt{H} \leqq \sqrt{H_{1}} \cup \sqrt{H_{2}} \cup \cdots \cup \sqrt{H_{k}}
$$

with $H_{i}$ pure and $H \nsubseteq H_{i}$ for all $i$. Since $\sqrt{H} \cap \sqrt{H_{i}}=\sqrt{H \cap H_{i}}$ we have

$$
\sqrt{H}=\sqrt{H \cap H_{1}} \cup \sqrt{H \cap H_{2}} \cup \cdots \cup \sqrt{H \cap H_{k}}
$$

and $H>H \cap H_{i}$. Now $\operatorname{dim} H>\operatorname{dim}\left(H \cap H_{i}\right)$ so by induction each $\sqrt{H \cap H_{i}}$ is a finite union of root sets of root reduced pure subgroups of $H \cap H_{i}$. Therefore, if we replace each $\sqrt{H \cap H_{i}}$ by its corresponding union, then the result follows.

If $H$ is a subgroup of a group $G$ we let core $H=\bigcap_{g \in G} H^{g}$ be the intersection of all $G$-conjugates of $H$. Thus core $H$ is the largest normal subgroup of $G$ contained in $H$. 
Lemma 5. Suppose $G$ is a linear group and

$$
G=\sqrt{H_{1}} \cup \sqrt{H_{2}} \cup \cdots \cup \sqrt{H_{n}}
$$

for pure subgroups $H_{i}$. Then for some subscript $j, G=\sqrt{\text { core } H_{j}}$, that is $G /$ core $H_{j}$ is torsion.

Proof. By Lemma 4 we may replace each $\sqrt{H_{i}}$ by a finite union of root sets of root reduced pure subgroups of $H_{i}$. Thus from the above we get

$$
G=\sqrt{W_{1}} \cup \sqrt{W_{2}} \cup \cdots \cup \sqrt{W_{t}}
$$

where for each $i, W_{i}$ is a root reduced pure subgroup of $G$ and $W_{i}$ is contained in $H_{i^{\prime}}$ for some $i^{\prime}$. Furthermore, by successively eliminating unnecessary $W_{i}$ 's in $\left(^{*}\right)$ we may assume that this union is irredundant. If $g \in G$, then conjugating $\left(^{*}\right)$ by $g$ yields for each $i$

$$
\sqrt{W_{i}} \subseteq G=\sqrt{W_{1}^{g}} \cup \sqrt{W_{2}^{g}} \cup \cdots \cup \sqrt{\overline{W_{t}^{g}}} .
$$

Thus since $W_{i}$ is root reduced we conclude that for each $i$ and $g$ there exists a subscript $s$ with $W_{i} \subseteq W_{s}^{g}$.

We show now by inverse induction on $\operatorname{dim} W_{i}$ that all G-conjugates of $W_{i}$ occur in the union $\left(^{*}\right)$. Suppose first that $\operatorname{dim} W_{i}$ is as large as possible. Then from $W_{i} \subseteq W_{s}^{g}$ and the maximality of $\operatorname{dim} W_{i}$ we have $W_{i}=W_{s}^{g}$ and $W_{s}=W_{i}^{g^{-1}}$ occurs in $\left(^{*}\right)$ for all $g \in G$. This starts the induction. Now suppose the result to be true for all $W_{k}$ with $\operatorname{dim} W_{k}>\operatorname{dim} W_{i}$. Let $g \in G$ and let $s$ be given by $W_{i} \leqq W_{s}^{g}$. If $W_{s}^{g}>W_{i}$ then $\operatorname{dim} W_{s}>\operatorname{dim} W_{i}$ so by induction $W_{s}^{g}$ occurs in (*). Thus since $\sqrt{W_{s}^{g}} \supseteqq \sqrt{W_{i}}$ we see that the $W_{i}$ term in $\left(^{*}\right)$ is redundant, a contradiction. Therefore, we must have $W_{s}^{g}=W_{i}$ so $W_{s}=$ $W_{i}^{g^{-1}}$ occurs in $\left(^{*}\right)$ and this fact follows.

Now $\left(^{*}\right)$ is a finite union so obviously each $W_{i}$ has only finitely many conjugates in $G$. Thus we can find a normal subgroup $\bar{G}$ of $G$ of finite index which normalizes each $W_{i}$. If $\bar{W}_{i}=\bar{G} \cap W_{i}$ then $\bar{W}_{i} \triangleleft \bar{G}$ and we have clearly

$$
\bar{G}=\sqrt[\bar{G}]{\bar{W}_{1}} \cup \sqrt[\bar{G}]{\bar{W}_{2}} \cup \cdots \cup \sqrt[\bar{G}]{\bar{W}_{t}} .
$$

Thus by Lemma 2 applied to the abstract group $\bar{G}$ we have $\bar{G}=\sqrt[\bar{G}]{\bar{W}_{i}}$ for some $i$ and hence since $G / \bar{G}$ is finite and $W_{i} \supseteq \bar{W}_{i}$ we obtain $G=\sqrt{W_{i}}$. Now $W_{i}$ has only finitely many conjugates and certainly for each $W_{i}^{g}, G=\sqrt{W_{i}^{g}}$. Thus since the intersections below are all really finite we have

$$
G=\bigcap_{g \in G} \sqrt{W_{i}^{g}}=\sqrt{\bigcap_{g} W_{i}^{g}}=\sqrt{\text { core } W_{i}} .
$$


Finally $W_{i} \subseteq H_{j}$ for some $j$ so core $W_{i} \subseteq$ core $H_{j}$ and $G=\sqrt{\operatorname{core} H_{j}}$. The result follows.

2. Centralizer subgroups. Let $G$ be a linear group so that $G \leqq L_{u}$. If $H$ is a subgroup of $G$, then we say that $H$ is a centralizer subgroup if $H=C_{G}(T)$ for some nonempty subset $T \subseteq L_{u}$.

Lemma 6. Let $H$ be a centralizer subgroup of $G$. Then $H$ is a pure subgroup and $G$ /core $H$ is a linear group.

Proof. Let $H=C_{G}(T)$. Then clearly $G \cap \hat{H}$ centralizes $T$ so $G \cap \hat{H} \subseteq H$ and $H$ is pure. If $g \in G$ then $H^{g}=C_{G}\left(T^{g}\right)$ so certainly core $H=C_{G}(S)$ where $S=\bigcup_{g \in G} T^{g}$. Let $\hat{S}$ denote the $L$-linear span of $S$. Then $\widehat{S}$ is a finite dimensional $L$-vector space and $G$ acts on $\widehat{S}$ by conjugation. Since clearly core $H=C_{G}(\hat{S})$ we see that $G /$ core $H$ is a linear group.

We now complete our group theoretic work.

Proposition 7. Suppose $G$ is a linear group and

$$
G=\sqrt{H_{1}} \cup \sqrt{H_{2}} \cup \cdots \cup \sqrt{H_{n}}
$$

where each $H_{i}$ is a centralizer subgroup of $G$. Then for some subscript $j, G /$ core $H_{j}$ is locally finite and hence $\left[G: H_{j}\right]=l . f$.

Proof. By the above lemma each $H_{i}$ is a pure subgroup of $G$ and thus Lemma 5 implies that for some $j, G /$ core $H_{j}$ is torsion. Then again by the above lemma $G /$ core $H_{j}$ is a periodic linear group and hence, as is well known, it is locally finite. Finally let $S$ be a finitely generated subgroup of $G$. Then $S$ (core $H_{j}$ )/core $H_{j}$ is a finitely generated subgroup of $G /$ core $H_{j}$ so

$$
S /\left(S \cap \text { core } H_{j}\right) \cong S\left(\text { core } H_{j}\right) / \text { core } H_{j}
$$

is finite. Since $S \supseteqq S \cap H_{j} \supseteqq S \cap$ core $H_{j}$ we have $\left[S: S \cap H_{j}\right]<\infty$ and hence $\left[G: H_{j}\right]=$ l.f.

Now we consider group rings.

Proof of the Theorem. Let $G, H$, and $K$ be as given and suppose first that $I=J K[G] \cap K[H] \neq 0$. Since $H$ is solvable and $I$ is a nonzero ideal of $K[H]$, results of [5] imply that $I \cap K[\ni(H)] \neq 0$. Thus 


$$
J K[G] \cap K[\ni(H)]=I \cap K[\ni(H)] \neq 0 .
$$

Now $\ni(H)$ is a normal solvable $\Delta$-subgroup of $G$ so by Theorem A of [2] there exist finitely many nonidentity elements $h_{1}, h_{2}, \cdots, h_{n}$ of $\exists(H)$ of order a power of $p$ with

$$
G=\bigcup_{1}^{n} \sqrt{\boldsymbol{C}_{G}\left(h_{i}\right)}
$$

Since $G$ is a linear group and $C_{G}\left(h_{i}\right)$ is a centralizer subgroup we conclude from Proposition 7 that for some $j,\left[G: C_{G}\left(h_{j}\right)\right]=1$.f. Now $h_{j} \neq 1$ has order a power of $p$ so we can choose $x \in\left\langle h_{j}\right\rangle$ to have order $p$. Since $\boldsymbol{C}_{G}(x) \supseteqq \boldsymbol{C}_{G}\left(h_{j}\right)$ we have $\left[G: \boldsymbol{C}_{G}(x)\right]=1$. f. and this half is proved.

Conversely suppose $x \in \ni(H)$ is given with $\left[G: C_{G}(x)\right]=1$.f. and suppose $x$ has order $p$. Then since $\ni(H)$ is a normal $\Delta$-subgroup of $G$ we see easily that the proof of the converse part of Theorem B of [2] carries over to prove the result that $J K[G] \cap K[\ni(H)] \neq 0$. Thus certainly $J K[G] \cap K[H] \neq 0$ and the result follows.

Proof of the Corollary. Let $G, H$, and $K$ be as above. If $H$ is nilpotent then by [5] the same result holds with $\ni(H)$ replaced by $\Delta(H)$. Thus we need only show that if $\Delta(H)$ has an element $y$ of order $p$ with $\left[G: \boldsymbol{C}_{G}(y)\right]=$ l.f. then we can also find such an element in $\boldsymbol{Z}(H)$.

Now $y \in \Delta(H)$ has finite order so $\langle y\rangle^{H}$, the normal closure of $y$ in $H$ has finite order. Moreover, since $\langle y\rangle^{H}$ is a finite nilpotent group generated by $p$-elements, it is a $p$-group. Since $H$ is nilpotent $\langle y\rangle^{H} \cap \boldsymbol{Z}(H) \neq\langle 1\rangle$ and we can choose $x$ to be an element of order $p$ in this intersection. Now $C_{G}(y)$ normalizes $\langle y\rangle$ and $H$ so it normalizes the finite group $\langle y\rangle^{H}$. Thus some subgroup of $\boldsymbol{C}_{G}(y)$ of finite index centralizes $\langle y\rangle^{H}$ and hence $\left[\boldsymbol{C}_{G}(y): \boldsymbol{C}_{G}(y) \cap \boldsymbol{C}_{G}(x)\right]<\infty$. By Lemma 1 (v) (i) of [3] we conclude first that

$$
\left[G: C_{G}(y) \cap \boldsymbol{C}_{G}(x)\right]=\text { l.f. }
$$

and then

$$
\left[G: C_{G}(x)\right]=\text { l.f. }
$$

The Corollary is proved.

\section{REFERENCES}

1. S. A. Amitsur, On the semisimplicity of group algebras, Michigan Math. J., 6 (1959), 251-253.

2. C. R. Hampton and D. S. Passman, On the semisimplicity of group rings of solvable groups, Trans. Amer. Math. Soc., 173 (1972), 289-301. 
3. D. S. Passman, Some isolated subsets of infinite solvable groups, Pacific J. Math.. 45 (1973), 313-319.

4. A. E. Zalesskii, On group rings of linear groups, Siberian Math. J., 12 (1971), 246-250.

5. — A semisimplicity criteria for the group ring of a solvable group, (in Russian), Doklady Akad. Nauk SSSR, (to appear).

Received March 23, 1972. Research supported in part by N.S.F. contract GP-29432. UNIVERSITY OF WISCONSIN, MADISON 


\section{PACIFIC JOURNAL OF MATHEMATICS}

\section{EDITORS}

D. Gilbarg and J. Milgram

Stanford University

Stanford, California 94305

R. A. Beaumont

University of Washington

Seattle, Washington 98105
J. DUGUNDJI* Department of Mathematics

University of Southern California Los Angeles, California 90007

RICHARD ARENS

University of California

Los Angeles, California 90024

\section{ASSOCIATE EDITORS}

E. F. BeCKenbach

B. H. NeUMaNN

F. WOLF

K. Yoshida

\section{SUPPORTING INSTITUTIONS}

UNIVERSITY OF BRITISH COLUMBIA

UNIVERSITY OF SOUTHERN CALIFORNIA

CALIFORNIA INSTITUTE OF TECHNOLOGY

UNIVERSITY OF CALIFORNIA

MONTANA STATE UNIVERSITY

STANFORD UNIVERSITY

UNIVERSITY OF TOKYO

UNIVERSITY OF NEVADA

UNIVERSITY OF UTAH

NEW MEXICO STATE UNIVERSITY

WASHINGTON STATE UNIVERSITY

OREGON STATE UNIVERSITY

UNIVERSITY OF OREGON

OSAKA UNIVERSITY

UNIVERSITY OF WASHINGTON

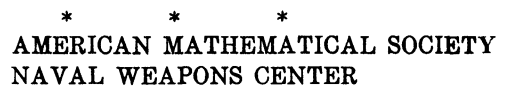

* C. DePrima will replace J. Dugundji until August 1974. 


\section{Pacific Journal of Mathematics}

\section{Vol. 47, No. $1 \quad$ January, 1973}

K. Adachi, Masuo Suzuki and M. Yoshida, Continuation of holomorphic

mappings, with values in a complex Lie group ....................

Michael Aschbacher, A characterization of the unitary and symplectic groups

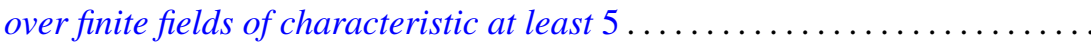

Larry Eugene Bobisud and James Calvert, Energy bounds and virial theorems for abstract wave equations....................................

Christer Borell, A note on an inequality for rearrangements ................

Peter Southcott Bullen and S. N. Mukhopadhyay, Peano derivatives and general

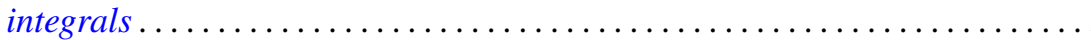

Wendell Dan Curtis, Yu-Lee Lee and Forrest Miller, A class of infinite dimensional subgroups of $\operatorname{Diff}^{r}(X)$ which are Banach Lie groups .........

Paul C. Eklof, The structure of ultraproducts of abelian groups ...............

William Alan Feldman, Axioms of countability and the algebra $C(X) \ldots \ldots \ldots$

Jack Tilden Goodykoontz, Jr., Aposyndetic properties of hyperspaces...........

George Grätzer and J. Płonka, On the number of polynomials of an idempotent algebra. II ...........................................

Alan Trinler Huckleberry, The weak envelope of holomorphy for algebras of

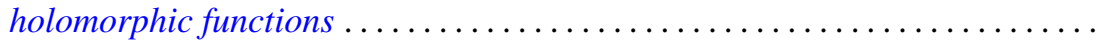

John Joseph Hutchinson and Julius Martin Zelmanowitz, Subdirect sum decompositions of endomorphism rings . . . . . . . . . . . . . . . .

Gary Douglas Jones, An asymptotic property of solutions of

$y^{\prime \prime \prime}+p y^{\prime}+q y=0$.

Howard E. Lacey, On the classification of Lindenstrauss spaces .

Charles Dwight Lahr, Approximate identities for convolution measure algebras.

George William Luna, Subdifferentials of convex functions on Banach

spaces.

Nelson Groh Markley, Locally circular minimal sets. .

Robert Wilmer Miller, Endomorphism rings of finitely generated projective modules

Donald Steven Passman, On the semisimplicity of group rings of linear

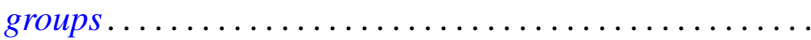

Bennie Jake Pearson, Dendritic compactifications of certain dendritic spaces.

Ryōtarō Satō, Abel-ergodic theorems for subsequences ...... .

Henry S. Sharp, Jr., Locally complete graphs. . .

Harris Samuel Shultz, A very weak topology for the Mikusinski field of

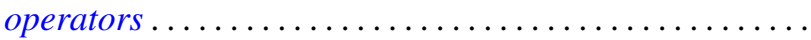

Elena Stroescu, Isometric dilations of contractions on Banach spaces ...

Charles W. Trigg, Versum sequences in the binary system ... . .

William L. Voxman, On the countable union of cellular decompositions of n-manifolds 